ESPAÑOLA Y DE LA UNIÓN EUROPEA

\title{
EL ARBITRAJE INTERNACIONAL COMO CAMINO HACIA UNA JUSTICIA JURÍDICO-PÚBLICA GLOBAL
}

\author{
HÉCTOR IGLESIAS SEVILLANO \\ Universidad Autónoma de Madrid \\ hector.iglesias@predoc.vam.es
}

Cómo citar/Citation

Iglesias Sevillano, H. (2018).

El arbitraje internacional como camino hacia una justicia jurídico-pública global. Revista de Administración Pública, 206, 291-318. doi: https://doi.org/10.18042/cepc/rap.206.10

\section{Resumen}

El presente trabajo aspira a construir un concepto de arbitraje internacional de inversiones como un fenómeno del derecho administrativo global, y no como un mero mecanismo jurídico-privado de resolución de controversias internacionales. Mediante una reconstrucción de la historia de la institución, intentamos demostrar cómo existe un proceso de evolución jurídica, desde un control extraestatal del poder público encomendado a la violencia y más tarde al derecho internacional, hasta un control jurídico-privado consentido por el propio Estado, mezcla esta de la que deriva la naturaleza híbrida de la institución, y que permite caracterizarla como derecho global. A partir de aquí, desarrollamos los aspectos del arbitraje internacional jurídicamente relevantes para el derecho público, algunos de los cuales son igualmente extensibles al arbitraje comercial.

\section{Palabras clave}

Arbitraje internacional de inversiones; derecho global; tratados de inversiones; ciudadano corporativo; función de control. 


\section{Abstract}

The present work aims at building a concept of International Investment Arbitration as a phenomenon of Global Administrative Law, and not as a mere mechanism of private law for the resolution of international conflicts. Through the reconstruction of the history of this institution, we try to show of the existence of a process of legal evolution, from the extra-State control of public power given to the hands of violence and later to International Law, to a system of control of private law consented by the State itself, a mixture that gives the institution an hybrid nature and that allows us to categorize it as Global Law. From this point, we will develop different aspects of International Arbitration which might be legally relevant for public law purposes, some of which might be extensible to commercial arbitration.

\section{Keywords}

International Investment Arbitration; Global Law; Investment Treaties; corporate citizen; control function. 


\section{SUMARIO}

I. LA IRRUPCIÓN DEL ARBITRAJE INTERNACIONAL DE INVERSIONES: AUGE Y CONSOLIDACIÓN. II. LAS POSICIONES CRÍTICAS: 1. La crítica norteamericana. El debate sobre la democratización. 2. La crítica hispanoamericana. Tentativas de regreso a Calvo. 3. La crítica europea: la STJUE en el caso C-284/16, de 6 de marzo de 2018 (Slovak Republic v Achmea BV). III. SÍNTESIS DEL DEBATE: EL ARBITRAJE DE INVERSIONES COMO PRIMER PASO EVOLUTIVO DE UNA JUSTICIA ADMINISTRATIVA GLOBAL. IV. LAS INSTITUCIONES DE ARBITRAJE INTERNACIONAL COMO CIUDADANOS CORPORATIVOS. EN CONCRETO, LA CONVENCIÓN ICSID DEL BANCO MUNDIAL. V. CONCLUSIÓN.

\section{LA IRRUPCIÓN DEL ARBITRAJE INTERNACIONAL DE INVERSIONES: AUGE Y CONSOLIDACIÓN}

El arbitraje internacional de inversiones es uno de los grandes hitos del derecho moderno, y podemos decir, sin duda, también del derecho global. No pocos estudios han intentado esclarecer su naturaleza y su relación con el derecho administrativo global ${ }^{1}$. El fenómeno en sí, desde un punto de vista

1 Solo a título de ejemplo, mencionamos aquí los que vamos a emplear en el análisis del fenómeno. En general, como estudio sobre el arbitraje internacional, es inevitable citar la obra de A. Redfern y M. Hunter (2015), International Arbitration (6 ed.), Oxford: OUP. Como bibliografía de estudio sobre las relaciones del arbitraje internacional de inversiones y el derecho administrativo global, aquí proponemos examinar las siguientes: A. A. Alshorbagy y A. Elattarl (2017), "The rise and fall of international administrative arbitration: An inquiry into the commerciality of international arbitration under Egyptian Law», International Review of Law, The Future of Alternative Dispute Resolution - A Qatari Perspective, Conference Proceedings 2017, 3; D. Bello Janeiro (2009), «Instrumentos internacionales en materia de arbitraje internacional, conciliación y mediación. El reconocimiento de resultados por parte de los estados», 2009 Workshop Internacional sobre ADR/ODRs. Construyendo puentes: marco jurídico y principios. Universitat Oberta de Catalunya (UOC), Internet Interdisciplinary Institute (IN3), 15 de septiembre de 2009; A. R. Brewer-Carías (2016), «El consentimiento del Estado al arbitraje internacional en la Ley 
histórico, está bien estudiado, y se aborda en la bibliografía aportada mucho mejor de cómo podamos hacerlo nosotros. No obstante, proponemos un breve análisis de este desarrollo, pero adoptando un punto de vista distinto del

de Promoción y Protección de inversiones de 1999 y sus vicisitudes», en J. RodríguezArana Muńoz y J. I. Hernández G. (2016), El Derecho Administrativo Global y el arbitraje internacional de inversiones. Una perspectiva iberoamericana en el marco del cincuenta aniversario del CIADI (págs. 129-196), Madrid: INAP; D. E. Childress (2013), «Does international investment law need administrative law? Responding to Jason Yackee», Harvard International Law Journal Online, 54, págs. 115-123; L. Ching-Lang (2014), Arbitration in administrative contracts: comparative law perspective, Paris: Institut d'Études Politiques de Paris; A. Gordillo y M. Brunos Dos Santos M. (dirs.) (2014), Tratado de derecho administrativo y obras selectas, Tomo 2, La defensa del usuario y del administrado, Buenos Aires: FDA; I. Granado Hijelmo (2005), «El arbitraje en Derecho Administrativo: algunas reflexiones sobre su fundamentación", Revista jurídica de Navarra, 39, págs. 39-56; J. I. Hernández G. (2016), «El CIADI en el marco del Derecho Administrativo Global: una visión desde el Derecho Administrativo Latinoamericano», en J. RodríguezArana Muñoz y J. I. Hernández G. (2016), El Derecho Administrativo Global y el arbitraje internacional de inversiones. Una perspectiva iberoamericana en el marco del cincuenta aniversario del CIADI (págs. 197-256), Madrid: INAP; I. Hernández G. (2017), «Regulación económica y arbitraje internacional de inversiones", Revista Electrónica de Direito, 1; B. Kingsbury y S. Schill (2009), «Investor-State Arbitration as Governance: Fair and Equitable Treatment, Proportionality and the Emerging Global Administrative Law», IILJ Working Paper n. 2009/6; S. Montt (2012), State Liability in Investment Treaty Arbitration: Global Constitutional and Administrative Law in the BIT Generation, Oxford: Hart Publishing; P. Navarro Rodríguez (2016), «El nuevo Derecho Administrativo global como lex administrativa en el arbitraje internacional de inversiones», Revista General de Derecho Administrativo, 42; A. Rajput (2012), «Advent of Investment Arbitration and Evolution of International Administrative Law», Indian Law Institute, 54, 2, págs. $232-$ 247; J. Rodríguez-Arana Muñoz y J. I. Hernández G. (2016), El Derecho Administrativo Global y el arbitraje internacional de inversiones. Una perspectiva iberoamericana en el marco del cincuenta aniversario del CIADI (págs. 129-196), Madrid: INAP; I. Salcedo Verduga (2009), «El arbitraje internacional y la contratación administrativo», revistajuridicaonline, 26, págs. 19-30; J. W. Yackee (2012), «Controlling the International Investment Law Agency», Harvard International Law Journal, 53, 2, págs. 392-448; G. van Harten y M. Loughlin (2006), "Investment Treaty Arbitration as a Species of Global Administrative Law», The European Journal of International Law, 17, 1, págs. 121-150; J. Watson Hamilton (2008), "Arbitration is not Administrative Law», abalawg.ca, 16 de agosto de 2008. Un importante y muy reciente trabajo sobre el nivel de intervención de los tribunales arbitrales en el control de la actividad jurídico-pública del Estado (aunque, a diferencia de nosotros, es desfavorable a esta) es J. Paine (2018), «Standard of Review (Investment Arbitration)», en H. Ruiz Fabri (ed.) (2019), Max Planck Encyclopedia of International Procedural Law, Oxford: OUP (obra de próxima aparición). 
habitual, en el que dicho desarrollo histórico es concebido como un camino hasta el derecho público global. Veámoslo.

El arbitraje internacional de inversiones no es en sí mismo — como no lo es ninguna institución jurídica - un objeto histórico de nacimiento espontáneo, sino una alternativa a un problema jurídico, político y económico, pero no de una sociedad o Estado en concreto, sino de naturaleza supranacional. Dicho problema es, ni más ni menos, la defensa de los intereses de los inversores particulares extranjeros (personas físicas o jurídicas, y con diferente nivel de poder económico) en los Estados destinatarios de las inversiones. Esto nos lleva ya, por sí solo, a todas las derivadas que, tanto a nivel teórico como histórico, el problema ha suscitado. Hay obviamente un problema de poder, el de un poder desigual que hay que equilibrar, sin que ningún sistema deba favorecer especialmente a ninguna de las partes, lo cual es difícilmente alcanzable. En la relación Estado-inversor vamos a ver cómo las relaciones de poder cambian constantemente, y cómo el arbitraje ofrece una (teórica) solución ${ }^{2}$.

Así, en el siglo xIx y a principios del xx, en la época de apogeo absoluto del Estado-nación, y a su vez el tiempo de máxima expansión del colonialismo, el problema de las relaciones Estado-inversor particular extranjero se reconoció como un problema que superaba las fronteras del Estado receptor de la inversión. El resultado será necesariamente un problema entre Estados. Además, resulta que, por causa del deber de respeto a la soberanía del Estado, el particular no nacional no puede ser tampoco objeto de derechos específicos frente a este que cercenen tal soberanía, sobre todo porque el sujeto individual físico o jurídico no es en ningún caso miembro de la sociedad internacional, ni tiene poder para reclamar dichos derechos si no es en las instituciones del propio Estado destinatario, que en cada caso proveerá un sistema judicial interno más o menos fiable para el inversor.

Para la teoría internacionalista desarrollada por las metrópolis, esta intervención del Estado nacional del inversor está sobradamente justificada, precisamente porque en la defensa de los intereses de sus inversores, el Estado está reafirmando sus propios derechos, ni más ni menos que asegurar que las obligaciones de terceros Estados en derecho internacional se cumplen respecto de sus nacionales ${ }^{3}$. Dichas obligaciones consistirían en un supuesto «estándar internacional» vigente en el derecho internacional público, que implicarían un determinado trato de mínimo respeto a los derechos de los nacionales ex-

2 Para una introducción histórica completa, véase Redfern (2015: 441) y también Hernández (2016: 197).

3 Así lo dijo, casi textualmente, la Corte Internacional de Justicia en el caso PanevezysSaldutiskis Railway (Estonia v Lithuania) [1939], PCIJ A/B 76, parágrafo 16, citada por Redfern (2015: 442). 
tranjeros, si bien fue configurado siempre en interés de las metrópolis ${ }^{4}$. Bien, es evidente que ahora la gran desventaja que suponía ser inversor ha virado radicalmente, siempre al amparo de un Estado fuerte, del que el inversor es nacional.

Este desequilibrio de poderes llevó a un modelo disparatado y bien conocido de presión por parte de las metrópolis a los Estados destinatarios de la inversión, la llamada "gunboat diplomacy», que consistía, literalmente, en enviar una flota de barcos de guerra a las costas del Estado destinatario para reclamarle las obligaciones pendientes en favor de los nacionales del Estado más fuerte. Cierto es que esta práctica fue superada por la llamada «doctrina Drago", formulada por el ministro argentino del mismo nombre durante una famosa "gunboat crisis», la de Venezuela en 1902, después consolidada en la Conferencia de La Haya en 1907. Esta es a su vez desarrollo de la famosa «doctrina Calvo», enunciada a su vez por el eminente jurista argentino Carlos Calvo, y que defendió que las reclamaciones de los inversores internacionales debían sustanciarse en un procedimiento interno dentro del Estado destinatario, de manera que se diera el mismo trato a los inversores extranjeros que se les daría a los nacionales 5 .

Esta revalorización de las competencias nacionales de los Estados receptores de inversiones, sobre la que volveremos más tarde, significó en su tiempo un viraje y una apuesta hacia modelos pacíficos de resolución de estos conflictos, aún hoy vigentes: la protección diplomática y el arbitraje de inversiones entre Estados. Este sistema, surgido también de la Segunda Conferencia de Paz de La Haya en 1907 en forma de un acuerdo llamado Convención sobre la Resolución Pacífica de las Disputas Internacionales, sigue enmarcado en una visión internacionalista del problema. La adopción del arbitraje es un paso decisivo sí, pero el inversor no tiene acción frente al Estado destinatario de la inversión.

La innovación fundamental se la debemos al justamente famoso Convenio sobre Arreglo de Diferencias Relativas a Inversiones entre Estados y Nacionales de Otros Estados de 1965, que pone en marcha el CIADI del Banco Mundial, una institución arbitral específicamente diseñada para resolver los conflictos arbitrales Estado-inversor, estando el inversor legitimado para ser parte (demandante) en el proceso. Esta legitimidad requiere de la previa firma

Analiza este estándar Hernández G. (2016: 212).

5 Así lo afirmó el propio Calvo en su obra (1868) Derecho Internacional teórico y práctico de Europa y América, París. Esta doctrina sería recogida en diversos textos internacionales hispanoamericanos, singularmente en la Primera Conferencia Internacional de Estados Americanos de 1889, donde la doctrina Calvo fue aprobada sin la concurrencia del voto a favor de los Estados Unidos de América. Para una cita de esta última, véase de nuevo Redfern (2015: 442). 
de un tratado internacional por parte del Estado destinatario y el Estado nacional del inversor, los famosos tratados bilaterales de Inversiones (BITs, por sus siglas en inglés), en el que el Estado destinatario admita que resolverá sus conflictos con los inversores por medio del arbitraje, o bien de la adopción de una Ley de inversiones interna, en la que el Estado reconozca que tales conflictos se derivarán a un arbitraje (para lo cual, huelga recordarlo, no hace falta el acuerdo con ningún tercer Estado). La naturaleza de este recurso, en el que tiene acción el inversor, pero que sigue teniendo cierta horizontalidad, pues es posible en la mayoría de los casos gracias a un BIT diseñado por el Estado de origen para proteger a sus propios inversores, ha hecho que se califique tal recurso jurídico como «diagonal» ${ }^{6}$.

En cualquier caso, lo que nos interesa a nosotros es el paso fundamental que se da cuando, admitiéndose un arbitraje privado para condenar a un Estado, y además sin la indispensable protección diplomática del Estado nacional del inversor (por más que esta exista normalmente a través de un BIT), se reconoce la supranacionalidad del problema jurídico, no su supuesta internacionalidad, y además se consolida su carácter híbrido. Esta es, al fin y al cabo, la visión que tenemos nosotros del arbitraje internacional de inversiones: es un paso más hacia el derecho de un mundo global, y por esencia, no puede estar definitivamente vinculado a uno u otro ordenamiento diseńado para regular un mundo pasado. Así, sostenemos firmemente que el arbitraje internacional de inversiones es nítidamente un fenómeno de derecho global, y su problemática no puede resolverse de forma plenamente satisfactoria por el derecho internacional público o por el derecho interno de un Estado en concreto.

\section{LAS POSICIONES CRÍTICAS}

\section{LA CRÍTICA NORTEAMERICANA. EL DEBATE SOBRE LA DEMOCRATIZACIÓN}

En la vivísima doctrina norteamericana, no han tardado en asociar el arbitraje internacional de inversiones con el derecho administrativo global. Tampoco han tardado en observar sus puntos débiles. En el fondo, se les reprocha falta de transparencia democrática y la imposición de los intereses de los inversores incluso sobre la iniciativa legislativa interna. En la forma, los arbitrajes internacionales de inversiones adolecen de faltas importantes en

6 Redfern (2015: 444). 
cuanto a transparencia y motivación, aunque ciertamente estos vicios son menores que en los arbitrajes entre partes privadas. El más singular trabajo que ha defendido esta posición ha sido el del profesor Yackee, quien ha abordado exhaustivamente la, por así decirlo, lista de problemas que se manifiestan en el arbitraje internacional de inversiones hoy ${ }^{7}$.

En primer lugar, según denuncia el autor, los órganos de arbitraje, singularmente del CIADI (en cuyo estudio se centra), cumplen una función cuasi judicial, y al mismo tiempo otra cuasi legislativa. Cumplen una función cuasi judicial porque, ciertamente, actúan como auténticos jueces del control de la actividad jurídico-pública. Al fin y al cabo, su función es realmente separar las reclamaciones de un inversor de los cauces internos de control de la actividad administrativa, para salvaguardar al inversor en cuestión tanto de los costes de litigar en el extranjero y en un sistema jurídico que desconoce como, especialmente, para evitar la parcialidad de estos órganos a favor del Estado demandado.

El autor, sin embargo, suscita una idea interesantísima cuando compara a los árbitros no con los jueces de lo contencioso-administrativo, de formación generalista, sino con los administrative law judges del derecho norteamericano, es decir, con tribunales administrativos, órganos que forman parte de la estructura de la propia Administración a la que juzgan y que están constituidos por miembros con mandato limitado en el tiempo, incluso estando sometidos al poder jerárquico de la propia Administración. Estos tribunales, largo tiempo rechazados en Europa continental, son ciertamente valorados en Estados Unidos como órganos técnicamente válidos y al mismo tiempo sensibles a los objetivos de las políticas públicas que juzgan. Este valor, el de comprender las políticas públicas que dan lugar a las reclamaciones de los inversores, es el que no se encuentra en los árbitros del CIADI, y lo que es más grave en opinión del autor, los árbitros no rinden cuentas ante ninguna institución democrática, y desde luego no aquella a la que condenan ${ }^{8}$.

Al mismo tiempo, el arbitraje cumple también una indudable función cuasi legislativa. Por un lado, parece claro que los árbitros, si bien ni crean ni están vinculados por ninguna «jurisprudencia» arbitral, ya que cada caso es diferente e independiente de cualquier otro, contribuyen, sin embargo, a la toma de decisiones legislativas de toda clase, desde interpretación normativa hasta concretización de normas muy abstractas. Este último supuesto es singularmente habitual ${ }^{9}$. Naturalmente, no puede sorprender que un jurista, más aún uno formado en el Common Law, vea en esta labor una acción legislativa.

Yackee (2012: 392).

Ibid.: 409.

Ibid.: 411. 
A esto hay que añadir la crítica que luego veremos se ha hecho en Hispanoamérica a los tribunales arbitrales, donde se les reprocha que condenen a los Estados por determinados cambios legislativos que son, en definitiva, expresión de su potestad legislativa. Sin embargo, esta crítica tiene ya menos fuerza: antes bien, el desarrollo de la responsabilidad del Estado-legislador, aunque limitada según los modelos (véase, sin ir más lejos, el español), permite que en el derecho público moderno se conciba la responsabilidad por el ejercicio de la potestad legislativa. Merece la pena hacer alguna referencia a la responsabilidad del legislador, precisamente porque esta es aún más necesaria en un derecho como el global, que se enfrenta a una actividad legislativa frenética protagonizada por multitud de entidades privadas. En este sentido, el desarrollo de toda responsabilidad del legislador, incluido el Estado democrático (incluso cuando lo es realmente), la vemos como positiva, y no como un rasgo a criticar.

Por último, es necesario hacer mención a una última función que se manifiesta en el arbitraje, o al menos en el CIADI, como es la cuasi ejecutiva. Efectivamente, aunque pueda parecer una apreciación menor, no hay que dejar de caer en la cuenta de las múltiples potestades que ejerce la propia institución, que sin duda puede determinar el curso de un caso. Por ejemplo, la Secretaría del CIADI administra todo el procedimiento arbitral, al tiempo que redacta borradores e informes, tanto sobre arbitrajes en concreto como sobre asuntos de interés común para el órgano (por ejemplo, sobre la conveniencia del desarrollo de un órgano de apelación arbitral $)^{10}$. A todo este trabajo habría que añadir toda una actividad de elaboración de estándares sobre materias diversas (procedimiento, transparencia, etc.) que realizan tanto la citada Secretaría como otras instituciones, como las reglas elaboradas por la International Bar Association. De estas reglas nos ocuparemos después bajo la rúbrica de soft law en el arbitraje internacional.

A estas críticas generales, el autor ha añadido otras más concretas. Es interesante ver cómo estas críticas son contestadas en un artículo posterior ${ }^{11}$. Así, para Yackee, el CIADI adolece de una importantísima falta de responsabilidad política democrática e impone sin una justificación suficiente un «derecho supranacional» no adoptado por ninguno de los Estados condenados, y esta situación se resolvería asimilando el organismo a una agencia estatal con responsabilidades e intervención política, que tuviera como misión fomentar la inversión (pero ¿una agencia de qué Estado?); para Childress, la crítica democrática no se sostiene, si se tiene en cuenta que son los propios Estados

10 Ibid.: 414.

11 Childress (2013: 115-123). 
miembros los que han adoptado el Convenio, y sobre todo si se tiene en cuenta que la mayoría de los Estados procesados están en la práctica lejos de cumplir con los estándares de una auténtica democracia (lo cual, aunque sea cierto en algunos casos, ni afirma ni desmiente la falta de control democrático del CIADI). Por otro lado, la cesión de potestades al CIADI que realizan los Estados está igualmente amparada en sus propias decisiones al adoptar el Convenio. Se mire por donde se mire, resulta claro para el autor que, si el CIADI no tuviera la configuración que tiene, restaría atractivo a los inversores no ya el hecho de acudir al CIADI para ejercitar sus reclamaciones, sino la inversión misma en el Estado destinatario ${ }^{12}$.

Efectivamente, el CIADI es un sistema imperfecto, como lo es a su vez el derecho global, que es la categoría jurídica en la que consideramos que se enmarca. Las críticas que se le dirigen, sobre todo a su falta de respaldo democrático, son muy justas, y los fallos democráticos de estas instituciones son a menudo serios. Las propuestas frente a esto, al menos las que vienen de planteamientos del derecho internacional público, hablan de incrementar el poder de los Estados demandados en el proceso, bien imitando la estructura orgánica y procedimientos de las Administraciones internas (la propuesta del modelo de la agencia estatal antes mencionada), bien directamente proponiendo soluciones puramente internacionalistas como, digamos, el control ex ante, que permitiría hacer observaciones al procedimiento arbitral tanto al Estado demandado como a terceros Estados que se declararan interesados en el procedimiento (interviniendo en el ejercicio de la función arbitral), o el control ex post, que consistiría en establecer comisiones constituidas por los Estados superiores al propio tribunal arbitral, que mediante la emisión de opiniones vinculantes para estos pudiesen alterar los propios laudos. El ejemplo propuesto en este caso es el de la Free Trade Commission (FTC) del Tratado NAFTA, que se compone de representantes de todos los Estados miembros y que emite esta clase de opiniones vinculantes para los tribunales arbitrales del Tratado, si bien es evidente que para adoptar esta solución es necesaria la actividad legislativa de un tratado internacional ${ }^{13}$.

Tampoco debe, sin embargo, idealizarse la opción contraria, la concepción acrítica del sistema vigente. Esta visión, a nuestro parecer, próxima más bien a una concepción iusprivatista, tampoco capta bien los avances del derecho global y es demasiado condescendiente con un poder que, como todos, debe ser sometido a control, y no debe asumirse su ejercicio tal y como lo conocemos como si fuese su formulación natural. El arbitraje internacional

12 Ibid.: 118.

13 Yackee (2012: 434). 
de inversiones, como cualquier otra institución jurídica, es un producto artificial de las necesidades de su sociedad y de su tiempo, por más que creamos que debe responder a ciertos elementos de justicia que podamos considerar inmanentes. Esta artificialidad, por cierto, es especialmente evidente en esta materia, donde el sistema actual ha pasado por tantas fases como hemos visto en los últimos doscientos años, y que en todo caso no se funda hasta que se firma el Convenio en 1965, y no se generaliza hasta los ańos noventa.

A nuestro parecer, el procedimiento de reflexión debe producirse más bien al revés: precisamente porque el problema es privado, debe quedar al margen de la Sociedad Internacional y la protección diplomática, y solo alcanzaría este nivel si por su magnitud —y aquí no vemos que quepan reglas generales - se convierte realmente en un problema estatal. $Y$ a su vez es un problema jurídico-público, pero no de derecho internacional, sino de derecho supranacional, porque el objeto del procedimiento será siempre el control de una entidad jurídico-pública (un Estado y cualquiera de sus órganos, agencias o representantes) por parte de un tribunal arbitral. El elemento jurídico público implica que este tribunal cumpla con los requisitos de tal control, tanto para la protección del sujeto que se enfrenta al Estado como para la defensa del interés público de este. Pero creemos firmemente que este objetivo no se logra de ninguna manera recurriendo a los modelos del derecho internacional público o privado, que no encajan plenamente con la problemática descrita, y por lo tanto sus soluciones serían inadmisibles.

A estos efectos hemos presentado esta importante crítica y contracrítica al arbitraje internacional de inversiones. En nuestra opinión, de momento, el arbitraje ha sobrevivido a duras argumentaciones en su contra. Veamos ahora si la recuperación de la doctrina Calvo puede o no hacernos cambiar de opinión.

\section{LA CRÍTICA HISPANOAMERICANA. TENTATIVAS DE REGRESO A CALVO}

Siguiendo con el esquema que hemos propuesto, vamos a continuar distinguiendo dos tipos de crítica: la protagonizada por la doctrina norteamericana, por un lado, que es la que acabamos de exponer, y la que se ha suscitado en Hispanoamérica, por otro. Esta polémica ha llegado a la retirada de ciertos países del Convenio CIADI, a pesar de que, de hecho, estos mismos habían mostrado gran interés en el desarrollo de los BITs pocos años antes. Veamos cómo sucede esto.

En primer lugar, hay que recordar el recorrido histórico que los Estados latinoamericanos realizan durante el siglo xIx y principios del $\mathrm{xx}$, precisamente en esta materia. Todos ellos son los protagonistas de algunos de los más sonados conflictos de inversiones y sobre todo de episodios de «gunboat diplomacy», siendo sin duda el más famoso el sucedido en Venezuela en 1902. 
Pues bien, como muy acertadamente ha estudiado Hernández, la doctrina Calvo derivó en un fortalecimiento del Estado y del derecho administrativo interno, en tanto que la doctrina del estándar mínimo de trato a los particulares, y su de algún modo supuesto sucesor, el propio Convenio CIADI, tienden a restringirlo ${ }^{14}$. Desde este punto de vista, es evidentemente lógica la adopción de la doctrina Calvo en el siglo xix y principios del xx: en la época de máximo apogeo del Estado-nación, los Estados latinoamericanos necesitan reafirmarse como tal. La doctrina Calvo les permite consolidarse, y hacer valer su propio sistema jurídico como un instrumento viable para regular sus relaciones con los inversores extranjeros. Es, por lo tanto, un refuerzo necesario de la estatalidad.

Más preocupante, y menos justificado, parece la recuperación de la doctrina Calvo en el siglo xxi. Efectivamente, ciertos países latinoamericanos, singularmente aquellos que componen el Acuerdo Ecoalba-TCP, cuyo art. 2 reproduce la doctrina citada ${ }^{15}$. Posteriormente estos mismos Estados denunciaron el Convenio CIADI, así Bolivia en 2007, Ecuador en 2009 y Venezuela en 2012. Singular resulta el caso de Venezuela, que de hecho había adoptado el sistema de Arbitraje internacional de inversiones no ya incorporándose al Convenio CIADI y firmando diversos BITs, sino mediante una ley, el Decreto Ley 356 de 13 de octubre de 1999, cuyo art. 22 remitía al arbitraje un conjunto muy amplio de contratos públicos del Estado y de sus entidades vinculadas, siempre que quedaran fuera del ámbito de iure imperi, procedimiento posible y admitido por los propios trabajos del Convenio CIADI ${ }^{16}$.

Pues bien, esta vuelta a la doctrina Calvo no puede ser, en nuestra opinión, bienvenida. Tal y como vemos el asunto, nos parece que la doctrina tuvo un muy importante papel en la defensa del derecho de los Estados latinoamericanos en el momento en que ese papel requería de ser desempeñado, es decir, en el momento de máximo auge del Estado-nación. Plantear, sin embargo, en el siglo XxI, en un contexto de globalización del derecho mediante su supranacionalidad, una doctrina que aísla el derecho interno de cualquier comunicación con el exterior, no creemos que sea positivo. La aplicación de la doctrina Calvo es ahora, digamos, extemporánea. No se trata de reivindicar la defensa de los valores o vigencia del derecho interno frente a potencias inversoras exteriores, pues por mucho que esas potencias existan, no basta con escapar del derecho internacional para huir de su influencia. Negarse a fenó-

14 Hernández (2016: 212). Véase también, desde una perspectiva latinoamericana, Salcedo (2009: 19).

15 Véase Hernández (2016: 211).

16 Han estudiado el caso de Venezuela, en particular, Brewer-Carías (2016: 129-196) y también Bello Janeiro (2009). 
menos como la ultraterritorialidad de muchas normas de derecho público, o a la vigencia del mismo derecho global, significa sencillamente aislarse, no ya de la sociedad internacional, sino de todo el avance del derecho supranacional descentralizado. No es conveniente para un Estado negar ni al fenómeno en sí ni a los contenidos que regula, y como estamos demostrando, una vía de acceso fundamental al derecho global son los propios tribunales de arbitraje.

Por otro lado, en el siglo XXI, no parece tan necesario reafirmar la autoridad del Estado como en el siglo xx. Por mucho que un Estado pudiera fortalecerse, jurídicamente hablando, el problema es el mismo: cada vez es más difícil encontrar un derecho estatal fuerte, y a su vez el derecho estatal es cada vez menos valorado por los destinatarios, y particularmente por aquellos que se mueven con facilidad a nivel global (hablamos fundamentalmente de personas jurídicas) y que confían cada vez más en una uniformidad jurídica que solo un derecho supranacional puede darles. Además, en ningún modo serviría renunciar al arbitraje internacional de inversores para evadirse del poder del derecho global, del que a día de hoy resulta difícil escapar. En definitiva, jurídicamente hablando, y sin entrar en cuestiones políticas de fondo, no entendemos de qué serviría a un Estado moderno llegar a un extremo de crítica tal al arbitraje internacional de inversiones como para renunciar a él completamente.

\section{LA CRÍTICA EUROPEA: LA STJUE EN EL CASO C-284/16, DE 6 DE MARZO DE 2018 (SLOVAK REPUBLIC V. ACHMEA BV)}

El arbitraje internacional de inversiones y los tratados bilaterales de inversiones han sufrido un reciente revés en el derecho europeo, a cuenta de la compatibilidad de este con las cláusulas del Tratado. La polémica se centra, en realidad, en los tratados bilaterales entre Estados miembros en los que es potencialmente aplicable el derecho europeo, en los que se discute si el debate puede sustraerse de los tribunales europeos (veremos más adelante qué se entiende por tales) para ponerse en manos de instituciones arbitrales internacionales. El Tribunal de Justicia de la Unión Europea responde a esta cuestión negativamente. La resolución es muy reciente y es pronto para llegar a conclusiones demasiado profundas, como tampoco es nuestra intención hacer una predicción a futuro de los posibles efectos que de ella puedan desplegarse. Pero, en cualquier caso, el asunto merece ser analizado con cierto detenimiento.

El rechazo del derecho europeo al Arbitraje de Inversiones en los últimos tiempos viene produciéndose por dos vías. Por una parte, mediante declaraciones públicas, fundamentalmente de la Comisión Europea, contrarias al pago de las condenas monetarias a las que los arbitrajes condenen a los Estados miembros en favor de demandantes que sean sujetos jurídico-privados 
nacionales de otros Estados miembros. Al cumplimiento de estos laudos condenatorios se les ha llegado a calificar como «ayuda de Estado» ${ }^{17}$.

El problema ha adoptado una dimensión considerable a causa de los procedimientos arbitrales internacionales iniciados contra Espańa tras la modificación del régimen de primas a las energías renovables, fundamentalmente mediante Real Decreto 1413/2014, de 6 de junio, por el que se regula la actividad de producción de energía eléctrica a partir de fuentes de energía renovables, cogeneración y residuos, la cual había sido publicada en el Boletín Oficial del Estado de 10 de junio de 2014, y contra la Orden IET/1045/2014, de 16 de junio, por la que se aprueban los parámetros retributivos de las instalaciones tipo aplicables a determinadas instalaciones de producción de energía eléctrica a partir de fuentes de energía renovables, cogeneración y residuos, publicada en el Boletín Oficial del Estado de 20 de junio de 2014.

Así, en estos casos, desde el ámbito del arbitraje han llegado dos condenas a día de hoy (se prevé que aumenten), los casos Eiser Infrastructure Limited y Energía solar Luxembourg del CIADI y Novenergía de la Cámara de Comercio de Estocolmo. Sin embargo, la Comisión Europea, reiterando su criterio en Micula, ha rechazado el pago de estos arbitrajes precisamente en virtud de la naturaleza de ayuda de Estado que tendría el pago. Así lo ha dicho la Comisión en su Decisión C(2017) 7384, dictada en el procedimiento de ayudas de Estado SA.40348 (2015/NN) - Spain, con carácter final. La Comisión concluye, entre otras cosas, que un órgano arbitral internacional (en este caso, creado en virtud de la Carta de la Energía) no es equiparable a un Tribunal de Derecho de la Unión en virtud de la jurisprudencia, y que, por lo tanto, en el supuesto de tener que aplicar el derecho europeo, no podría plantear la pertinente cuestión prejudicial ante el TJUE (fundamento 162) y que, en todo caso, cualquier conflicto con el Tratado (a estos efectos, la Carta de la Energía)

17 Así, la Comisión Europea ya ha impedido antes a un Estado miembro el cumplimiento de un laudo arbitral con este argumento. Por ejemplo, así lo hizo en su Decisión C(2014) 3192 final de la Comisión, de 26 de mayo de 2014, en el asunto sobre ayudas de Estado SA.38517 (2014/NN) — Micula v. Rumanía (laudo arbitral del CIADI), por la que se ordena a Rumanía la suspensión de cualquier actuación con vistas a la ejecución o a la aplicación del laudo arbitral de 11 de diciembre de 2013, por considerar que el cumplimiento constituiría una ayuda de Estado. El caso se produjo en el contexto de un tratado bilateral sobre inversiones entre Rumanía y Suecia, en una situación por lo tanto asimilable a la de la sentencia, que explicaremos inmediatamente. La posterior condena a la recuperación de la ayuda de Estado se produjo en la Decisión 2015/1470 de la Comisión de 30 de marzo de 2015. Esta Decisión se encuentra actualmente recurrida ante el Tribunal General de la Unión (Recurso interpuesto el 2 de septiembre de 2014 —Micula y otros/Comisión (asunto T-646/14). 
debería resolverse en favor de la primacía del derecho de la Unión, y por lo tanto esta carta no es aplicable entre Estados miembros (fundamento 163). Esta es, exactamente, la conclusión del caso Slovak Republic v. Achmea BV.

El planteamiento del TJUE en este caso (apartándose por cierto de la opinión del abogado general) no difiere sustancialmente del punto de vista planteado por la Comisión, aunque sí del sentido con el que el órgano judicial que planteó la cuestión prejudicial, el Bundesgerichtshof de Alemania, pretendía abordarla. Para el tribunal federal alemán, el arbitraje entre una empresa de seguros holandesa y el Estado de Eslovaquia amparado en un tratado bilateral de inversiones y cuya celebración se produjo en Frankfurt, no se vería entorpecido por el derecho europeo al no violar ningún precepto de los tratados, si bien plantea la cuestión prejudicial precisamente a falta de otros pronunciamientos del tribunal en la misma materia.

Las violaciones alegadas por Eslovaquia en su intento de anular el laudo y de cuya viabilidad jurídica duda el tribunal federal alemán fueron los artículos 18, 267 y 344 del Tratado de Funcionamiento de la Unión Europea. Junto a la prohibición de discriminación por nacionalidad y la discusión sobre la exclusión de los tribunales arbitrales del concepto de Tribunales Europeos por no poder plantear cuestión prejudicial, Eslovaquia alegaba también la incompatibilidad de la solución arbitral de controversias con el compromiso de los Estados de no someter la aplicación o interpretación de los tratados a un procedimiento distinto del previsto en el mismo. A ninguno de los tres argumentos daba el Bundesgerichtshof credibilidad suficiente como para poder desplazar el Tratado Internacional de Inversiones, y menos aún al último, que no parece ni aplicable al supuesto de hecho, al referirse solo a la interpretación y aplicación de los tratados.

Sin embargo, la conclusión del Tribunal de Justicia de la Unión Europea es muy diferente. En primer lugar, toda la respuesta se fundamenta en la autonomía del derecho de la Unión, principio cuya expresión jurídica más prominente es precisamente el art. 344 del Tratado de Funcionamiento, con lo cual este precepto sí que se declara de entrada aplicable (FJ 32). Por otro lado, el Tribunal de Justicia hace suyo un argumento ya expresado por la Comisión, como es la falta en los tribunales arbitrales internacionales de la naturaleza jurídica de los tribunales de la Unión. En términos de derecho europeo esto significa que estos órganos no pueden presentar cuestión prejudicial ante el Tribunal de Justicia del art. 267 del Tratado de Funcionamiento (FJ 46) y esto supone una amenaza a la propia autonomía y uniformidad interpretativa del ordenamiento de la Unión (FJ 35). Además, de forma un tanto sorprendente, aparece un argumento del Tribunal según el cual la naturaleza de un arbitraje de inversiones sería diferente de un arbitraje comercial, pues no depende exclusivamente de la autonomía de la voluntad de los contratantes sino que deriva de un tratado internacional 
e implica que el tribunal arbitral puede tener que aplicar derecho público (incluido por supuesto el europeo) (FJ 55). La incompatibilidad del tratado con el derecho europeo se debería no solo al principio de confianza mutua entre los Estados miembros (que acuerdan un Tratado Bilateral de Inversiones al margen del derecho de la Unión, aunque este sea anterior a la integración de uno de los dos Estados en aquella, como era el caso), sino también a la quiebra de la uniformidad y autonomía del ordenamiento jurídico de la Unión (FJ 58). Como consecuencia de todo esto, el Tribunal de Justicia de la Unión Europea entiende que la conjunción de los arts. 267 y 344 del Tratado de Funcionamiento de la Unión Europea impide la eficacia de un Tratado de arbitraje internacional entre dos Estados miembros.

Como ya hemos dicho más arriba, no es nuestra intención verter una opinión definitiva sobre esta decisión, cuyos efectos aún están por desplegarse. Es previsible, no obstante, que esta sea el inicio de una jurisprudencia que tenderá a consolidarse. Y es innegable que representa un punto de vista compartido en las instituciones europeas, especialmente por la Comisión. No es ni mucho menos una opinión particular del Tribunal de Justicia. El paso hacia la exclusión del arbitraje internacional de inversiones entre Estados miembros parece decidido.

Otra cosa es el juicio que merezca este camino. Naturalmente, la peculiar posición que el arbitraje internacional de inversiones ha adquirido en el derecho global, como mecanismo de control del poder público por parte de entidades privadas, y las enormes posibilidades que esta vía de ejercicio de la función de control abren tanto para el control de la Administración estatal y la homogeneización de los principios jurídicos del Estado de derecho a nivel global, como para el control de las normas supranacionales, pueden hacer dudar de la necesidad de una doctrina como esta.

Ciertamente, las intenciones de las instituciones de la Unión, y en particular del Tribunal de Justicia, con esta decisión parecen loables. La consolidación del ordenamiento jurídico europeo (de cuya naturaleza ya pueden caber pocas dudas) y la defensa de su autonomía y eficacia son principios de gran importancia para la Unión. Lo preocupante, en realidad, no es tanto la preservación del ordenamiento comunitario de la influencia de agentes externos, sino la evidencia de que la autonomía del ordenamiento jurídico, entendida en el sentido tradicional que ha tenido en el Estado-nación en los siglos XIX y XX es un concepto difícil de aplicar a la Unión, y lo que es más, en un contexto jurídico global corre el riesgo de aislar el derecho europeo de las influencias externas de otros ordenamientos jurídicos, tanto estatales como supranacionales.

Por otra parte, centrándonos en los términos de la decisión judicial, el Tribunal de Justicia de la Unión Europea nos ofrece de nuevo una de sus ca- 
racterísticas resoluciones, de la que conocemos al ponente, el magistrado Tizzano, si bien no tenemos acceso a posibles votos particulares. Por otra parte, y como también es habitual en este tribunal, los argumentos jurídicos resultan de una claridad que hace que parezcan evidentes, si bien mediante este proceder perdemos cierta noción del debate que subyace a tales conclusiones, cuando es evidente que esta es, como tantísimas otras, una cuestión jurídica en la que existe una amplia gama de grises. Distinta impresión nos da la opinión del abogado general Wathelet, así como el planteamiento de la cuestión por parte del Bundesgerichtshof alemán, aunque sus argumentos no fueron adoptados.

Así, por ejemplo, parece que la incompatibilidad del arbitraje internacional de inversiones con el principio de autonomía del derecho de la Unión es tanta como la que tiene con cualquier ordenamiento estatal, en el que el Estado tiene que renunciar a litigar en sus propios tribunales para satisfacer las necesidades de los inversores extranjeros. Sin embargo, esto es así por razones históricas, que ya hemos explicado. Por otra parte, la aplicación del derecho europeo en un tribunal arbitral se produce en las mismas condiciones que la aplicación de cualquier otra legislación estatal que hayan podido elegir las partes, o de un tratado internacional que regule una relación jurídica, como puede ser el propio Tratado Bilateral de Inversiones o la Carta de la Energía, donde tampoco existe recurso a los órganos judiciales del Estado cuyo ordenamiento jurídico ha sido elegido como ley aplicable al fondo de la cuestión, para asegurar la unidad de dicho ordenamiento. Por supuesto, la estipulación del arbitraje por medio de un tratado internacional es una situación distinta de facto que la de un acuerdo arbitral comercial, pero precisamente este mecanismo ha servido para perfeccionar el control de la actividad del Estado. Incluso en términos de política legislativa resulta difícil entender esta decisión, dada la amplia aceptación que tienen los tratados de inversiones tanto dentro como fuera de la Unión, y sobre todo teniendo en cuenta que muchos de los demandantes en litigios arbitrales en virtud de tratados de inversiones entre Estados miembros cuentan en realidad con capitales extracomunitarios, de manera que se niega el acceso al procedimiento arbitral a sujetos solo nominalmente ciudadanos europeos y previsiblemente se incrementan los costes de su inversión.

En definitiva, el Tribunal de Justicia de la Unión Europea ha abierto con Achmea $B V$ un panorama complejo e incierto, y desde luego determinante para el futuro de la Unión en muchos sentidos, tanto político-jurídico como probablemente económico. En todo caso, quedaremos a la espera del desarrollo de esta jurisprudencia y su implantación práctica. 


\section{SÍNTESIS DEL DEBATE: EL ARBITRAJE DE INVERSIONES COMO PRIMER PASO EVOLUTIVO DE UNA JUSTICIA ADMINISTRATIVA GLOBAL}

Pues bien, por seguir con el símil vital que hemos propuesto más arriba, creemos estar en condiciones de afirmar que el arbitraje internacional de inversiones, llegados a este punto, ha sobrevivido a sus críticos. Ahora bien, lo que es evidente es que estas críticas al menos sí que han puesto de relieve debilidades serias de la regulación, tanto del arbitraje de inversiones en general, como del CIADI en particular.

Para resolver estos ciertamente graves problemas, una importante línea crítica (sobre todo del derecho anglosajón) ha acudido ya a la solución que aquí proponemos: la categorización de estos arbitrajes como derecho administrativo global. Por otro lado, y en la extensión que veremos en el epígrafe siguiente, los principios de derecho global pueden extenderse no solo al arbitraje de inversiones, sino a todo el arbitraje, incluido el que es estrictamente de derecho privado.

En un texto escrito por B. Kingsbury y S. Schill, ambos afirman con acertada contundencia que el arbitraje de inversiones no es solamente un mecanismo de resolución de conflictos, sino un instrumento de gobernanza global que implica el ejercicio de un poder arbitral (agregamos nosotros, de control) en el «espacio administrativo global» ${ }^{18}$. No entraremos en este momento en el debate sobre si dicho espacio existe literalmente como tal. Parece exagerado hablar de que exista realmente un ámbito jurídico global en el que se reconozcan las normas de derecho administrativo global. Este planteamiento refleja la propuesta de este autor sobre la existencia de un hecho social global de reconocimiento de estas normas, pero dejamos aquí constancia de las dudas sobre la aplicabilidad real de esta teoría: en nuestra opinión, la eficacia de las normas supranacionales depende de su reconocimiento por parte del sujeto al que van a ser aplicadas. Lo que es más, tal propuesta nos obliga a reconocer una suerte de espacio físico donde se reconoce el derecho global, lo cual como bien sabemos es imposible, porque el derecho global por definición es esencialmente supranacional, lo que significa que no se proyecta sobre un territorio en concreto, ni tiene eficacia directa en él.

18 Kingsbury Schill (2009). En España, y presentando un trabajo muy innovador en nuestro ordenamiento jurídico, sigue a Kingsbury P. Navarro Rodríguez (2016), quien además adapta el planteamiento del derecho administrativo global también a otros problemas típicos del derecho administrativo español, como el principio de unidad de mercado nacional o la responsabilidad patrimonial de la administración (RPA). 
Decimos todo esto para aclarar ya desde el principio que, en nuestra opinión, el derecho global tiene en realidad una eficacia casuística y tópica, que requiere un reconocimiento concreto, y por lo tanto la aplicación de las propuestas solo cabe en el caso, e impactará más bien influyendo en el derecho público que esté en juego en cada supuesto, y no en un orden jurídico-público global que, como sistema, aún no conocemos. Por lo tanto, asumido que este poder de control en manos de tribunales arbitrales tiene una eficacia casuística, podemos entrar a valorar - y compartir - las críticas de estos autores.

En primer lugar, el foco se sitúa necesariamente en una cláusula de las más comunes en los BITs, convertida casi en indispensable: la cláusula de "fair and equitable treatment», que compromete al Estado firmante a dar un trato justo y equitativo al inversor nacional extranjero. Varias observaciones caben aquí. En primer lugar, parece evidente que esta cláusula es una reproducción del estándar internacional patrocinado por las metrópolis desde el siglo XIX, y como tal se ha interpretado inicialmente en los laudos arbitrales. Parece que entonces serviría para proteger aquellos valores (derechos de propiedad, personalidad y capacidad jurídica, vida e integridad física, libertad religiosa, igualdad de trato, derecho de acceso a la jurisdicción) ${ }^{19}$.

Sin embargo, se observa ya en estos momentos de evolución de la interpretación de esta cláusula que aparecen ciertos elementos de derecho público global, si bien el paso del estándar internacional al supranacional parece dudoso y difícil de identificar ${ }^{20}$. Lo que nos interesa a nosotros, más bien, no es tanto si una exigencia, por ejemplo, de respeto a los derechos procesales mínimos del inversor en el Estado demandado se justifica en principios de derecho internacional o supranacional, sino que estos derechos pueden ser controlados (y dicho Estado condenado) por un tribunal arbitral. Siendo esto así, no nos importa si la teoría sobre el fondo que se aplique en el control se funda en principios consolidados de derecho internacional o en novedosos análisis de global governance, porque lo que es derecho global no es solo el contenido jurídico de fondo sino el procedimiento, es decir, la atribución de la función jurídico-pública de control a un órgano arbitral no estatal.

Vamos a detenernos un momento para explicar mejor lo que queremos poner sobre la mesa. El planteamiento de estos autores ya es de por sí muy novedoso: los tribunales arbitrales cumplen una función de gobernanza global, de lo que se sigue que están desarrollando mecanismos de control (es decir, juicios jurídicos de fondo) que no pertenecen al ordenamiento jurídico de un

19 Sobre este contenido del estándar, véase A. H. Roth (1949), The minimum standard of international law applied to aliens, La Haya, págs. 128-191, cit. por Hernández (2016: 216).

20 Kingsbury y Schill (2009: 9). 
Estado, sino al orden jurídico global. Pues bien, esto sería derecho administrativo global. Luego, en el caso concreto de las cláusulas de "fair and equitable treatment", dado que estas se adoptan en tratados internacionales, son herederas de un acervo teórico del derecho internacional público (y por lo tanto de las relaciones entre Estados en la sociedad internacional, que es muy distinta del nivel supranacional, como ya sabemos) y dado que incluso se aplican a casos, a veces bastante burdos, de violación de los derechos de los ciudadanos extranjeros, digamos procesales por ejemplo, en los que la clásica teoría del derecho internacional basta para vislumbrar la violación y condenar al Estado infractor, entonces en estos casos no está claro que se haya desarrollado aún un auténtico derecho global, sino que más bien parece que se está aplicando la misma teoría tradicional, y los estándares de gobernanza global están por formar.

Este último planteamiento, sin embargo, es el que impugnamos. Porque sin duda los autores tienen razón en su análisis del derecho aplicable: con el derecho internacional vigente es suficiente para condenar por la mayoría de las infracciones de «fair and equitable treatment». Aquí el derecho global se está formando lentamente, a medida que se comprende que la protección del inversor no deriva tanto de un deber de un Estado respecto de otro y sus nacionales, sino de un nivel global de protección que se debe a todos los sujetos por igual en toda clase de relaciones jurídicas, y que debe tener en cuenta los múltiples intereses de todas las partes afectadas por cada medida, incluso aunque no estén representadas en el proceso en cuestión. Esta es, al menos, nuestra visión de cómo debe ser una justicia global.

Pero es que aunque esta justicia global no se haya realizado verdaderamente en el plano sustantivo, sí que se ha presentado en el plano formal, en la medida en que, en la resolución del caso, ha intervenido un tribunal arbitral privado (es decir, no vinculado a ningún poder público) y se ha dictado un laudo que no tiene en sí mismo el carácter de norma pública y no requiere la intervención del propio Estado, por más que para ejecutarlo haya sido necesario el consentimiento del propio Estado mediante un tratado, precisamente porque, como sabemos, en el derecho global es indispensable el consentimiento de todas las partes para que sus normas (en este caso, las normas de arbitraje de turno, el laudo, etc.) tengan eficacia.

Luego podemos concluir que, en primer lugar, por mucho que el tribunal arbitral aplique derecho internacional — cosa discutible —, el mero hecho de que el Estado sea controlado por un tribunal arbitral privado ya nos sitúa en el ámbito del derecho global. Y en segundo lugar, cuando el Estado acepta un acuerdo de arbitraje o la remisión futura a este en un tratado o en una ley interna, y cuando acepta la ejecución del laudo mediante un tratado internacional, no está dando un barniz jurídico-público ni a las normas de arbitraje que acepta (que son normas internacionales) ni al propio laudo (que es una 
norma privada internacional), sino que está dando el necesario consentimiento que todas las partes deben dar en el derecho global para que estas normas sean eficaces respecto de sí mismos.

Cosa distinta es que estas normas tengan su propio procedimiento de legitimación en el derecho del Estado. En este sentido, podemos afirmar que el arbitraje internacional se rige por regulaciones que son normas privadas internacionales (aunque las aprueben organizaciones internacionales constituidas por Estados) y dicta laudos que son en sí mismos normas privadas internacionales, y que en ambos casos estas normas tienen un procedimiento de legitimación propio que consiste en un procedimiento judicial de ejecución del laudo. Son, por lo tanto, un supuesto especial de norma privada internacional.

Otras propuestas de ambos autores son también atractivas, si bien sin duda muy volcadas en las preferencias del derecho anglosajón. La predictibilidad y consistencia de las decisiones del Estado infractor, la defensa de las expectativas legítimas del inversor, el respeto al procedimiento y la transparencia y proporcionalidad son líneas básicas de la propuesta ${ }^{21}$. Estas líneas maestras deberán ser seguidas tanto por el tribunal arbitral en el control de la actividad administrativa del Estado demandado como por los sujetos (Estados e inversores) que participan en el arbitraje en el control del propio tribunal arbitral, que deberá ejercerse mediante la configuración de sus reglas internas o reglas de referencia aplicables, y también, aunque en la medida en que quepa, mediante el control interno de la ejecución del laudo arbitral.

Lo que hemos querido resaltar en el presente epígrafe es que, aunque criticable, el arbitraje es el mecanismo de resolución de conflictos del derecho global. Hemos delimitado algunos de estos mecanismos de control, si bien un estudio exhaustivo de las propuestas no puede realizarse aquí, y debe remitirse a la doctrina citada. Ahora bien, quedan interrogantes abiertos. Por ejemplo, está claro que nos hemos centrado en el arbitraje internacional de inversiones porque es, dentro de todos los sistemas supranacionales de resolución de conflictos, el señalado por toda la doctrina internacional como la gran manifestación de derecho administrativo global. Hemos concluido que, ciertamente, lo es, y lo hemos incardinado en nuestro propio concepto de derecho global:

21 Kingsbury y Schill (2009: 10). Un tratamiento específico sobre la transparencia lo ha ofrecido C. Marian (2010), «Balancing Transparency: The Value of Administrative Law and Mathews-Balancing to Investment Treaty Arbitrations», Pepperdine Dispute Resolution Law Journal, 10, 2, págs. 275-301. Sobre la transparencia, téngase en cuenta la firma del Convenio de Naciones Unidas sobre la Transparencia en el arbitraje internacional de inversiones, firmado en Nueva York en 2014 (la «Mauritius Convention on Transparency»). Al mismo tiempo, y en la misma línea de análisis, véase Van Harten y Loughlin (2006). 
sabemos que es supranacional, porque se sitúa en un plano no territorial ni propio de las relaciones entre Estados; sabemos que es híbrido, porque ejerce un control privado de la Administración y de la actividad jurídico-pública del Estado, y ya hemos visto que, polémicamente, también puede ejercitar un control (o según algunos, potenciación) sobre las propias políticas de fondo que subyacen a esas decisiones jurídicas. El arbitraje internacional de inversiones ejerce una potestad de control privada sobre sujetos jurídico-públicos y como tal es, sin duda, derecho administrativo global.

Pero ahora vamos a virar el rumbo y ampliar nuestras miras. Más allá del arbitraje de inversiones se encuentra el mundo amplísimo del arbitraje jurídico-privado, normalmente llamado commercial en inglés, aunque de hecho su contenido material ya ha superado las barreras del derecho mercantil22. Este es un terreno inexplorado para el iuspublicista. Sin embargo, como vamos a demostrar, todo el arbitraje internacional, y no solo el de inversiones, es derecho global, en la medida en que todo tribunal arbitral ejerce una potestad jurídico-pública, la jurisdicción, y también la normativa global, como hemos dicho ya, mediante la emisión de laudos. Ahora bien, dado que en estos casos el arbitraje internacional se limita a las relaciones entre particulares, y no al Estado, en el arbitraje internacional común volveremos a las reglas más flexibles que hemos propuesto a lo largo de todo el trabajo, permitiendo a los sujetos obligados por las normas arbitrales (tanto las partes del arbitraje como los propios árbitros) el amplio margen de actuación que ya tienen, e incluso tratando de ofrecerles una protección jurídica mayor dentro del Estado.

\section{LAS INSTITUCIONES DE ARBITRAJE INTERNACIONAL COMO CIUDADANOS CORPORATIVOS. EN CONCRETO, LA CONVENCIÓN ICSID DEL BANCO MUNDIAL}

Comencemos entonces con los rasgos del derecho administrativo global que, en nuestra opinión, son predicables de todos los arbitrajes internaciona-

22 Así, Redfern y Hunter (2015: 11) ya anuncian en su obra que, si bien en ediciones anteriores dedicaron gran esfuerzo a la delimitación del concepto de commercial, y a pesar de que este se encuentra aún en los tratados (singularmente, en la Convención de Nueva York) y que es utilizado por algunos Estados para delimitar el ámbito de aplicación de sus normas internas de arbitraje internacional y arbitraje interno, su importancia real en el mundo del arbitraje ha disminuido drásticamente en los últimos años al ampliarse el ámbito del propio arbitraje internacional, y como consecuencia reducen también drásticamente su tratamiento en la obra. De ahí que nosotros tampoco le prestemos especial atención al supuesto requisito del arbitraje de ser «comercial». 
les. El más obvio de ellos es, probablemente, el de los sujetos. Como es bien sabido, el arbitraje internacional se divide en dos modelos: el arbitraje ad hoc y el arbitraje institucional. En el primero de ellos las partes gestionan la organización de su propio arbitraje, recurriendo a la aplicación de ciertas reglas internacionales, por ejemplo, por citar la más famosa de ellas, UNCITRAL, que enseguida vamos a caracterizar como una norma privada internacional.

En el segundo, el arbitraje institucional, es una organización la que se encarga de proporcionar no solo los árbitros, sino también todo el soporte normativo (cuentan con sus propios reglamentos internos, que son obviamente normas privadas internacionales) y de gestión (las secretarías, de las que hablábamos más arriba). Estas organizaciones son sobradamente conocidas: la International Commercial Chamber (ICC), fundada en París en 1923, la American Arbitration Association (AAA), la London Court of International Arbitration (LCIA) y, desde luego, el propio International Centre for the Settlement of Investment Disputes (ICSID, CIADI por sus siglas en español), además de múltiples cámaras de comercio a nivel mundial. Cualquiera de estos ejemplos serviría para fundamentar nuestra teoría: las organizaciones de arbitraje institucional son ciudadanos corporativos.

Con el concepto de ciudadano corporativo como entidad que ejerce la potestad de facto de emitir normas supranacionales, ciertamente no es difícil incluir a estas instituciones. En primer lugar porque, deliberadamente, hemos dejado el concepto muy abierto. No podemos saber a priori cómo es un sujeto que emite autónomamente normas internacionales. No hay una forma jurídica, un objeto social, ni siquiera un entorno social o económico concreto, ni mucho menos una atribución de potestad normativa explícita o tácita, que nos permita identificar a estos sujetos. Tampoco hay una lista. El ciudadano corporativo puede aparecer en forma de sociedad mercantil, organización internacional, agencia estatal con gran autonomía de actuación respecto de su Estado o reunión informal de sujetos con poder público. Es un sujeto que, en su forma, muta constantemente, pero es consistente con una forma de actuar y el ejercicio de una potestad normativa. Además, téngase en cuenta que la teoría del ciudadano corporativo la construimos para justificar precisamente el ejercicio de la potestad normativa, pero esto no significa que el ciudadano corporativo ejerza exclusivamente esta función, sino muy al contrario: es un sujeto de relaciones jurídicas de todo tipo que, en ciertos momentos, adopta la posición de autor de normas privadas internacionales.

Lo que hay, por tanto, es simplemente una práctica muy extendida de generar normas que los sujetos pueden aplicarse a sí mismos, adoptándolas de diferentes formas. Las normas, sin embargo, preexisten a cualquier contrato y se exhiben como válidas frente a todos a priori, por mucho que eso no implique su eficacia directa. Lo contractual serán las cláusulas que las partes hayan 
pactado, pero el efecto del contrato que se remite a la aplicación de una norma privada internacional es sencillamente prestar el consentimiento indispensable para que la norma le sea aplicada a la parte que lo presta.

Siendo esto así, entendemos que no cabe confusión posible de las normas privadas internacionales con cláusulas contractuales, y por lo tanto no cabe escudarse simplemente en que normas como las de una institución arbitral han sido contractualmente admitidas por las partes: lo han sido, pero lo que han hecho las partes es prestar su consentimiento recepticio para que se les apliquen a sí mismas, y en la medida en que son normas cabe someterlas a control. De ahí la importancia que, consideramos, tiene admitir el carácter de ciudadano corporativo que tienen las instituciones arbitrales.

Por el momento, se puede decir poco más de estos ciudadanos corporativos, aunque es cierto que creemos que el arbitraje es un gran campo de pruebas. Decimos en el sentido de que, en el futuro, una vez que se haya asumido la realidad del ciudadano corporativo y sus normas, pueda ser común la extensión de potestades administrativas de policía y regulatorias a estos sujetos. Ya hemos dicho en alguna ocasión que, por ejemplo, no debería sorprendernos ver en el futuro inspecciones o sanciones a estos sujetos. Para realizar esta tarea, complicada desde luego, solo vemos tres opciones.

La primera sería que estas facultades fueran ejercidas por los propios Estados en los que van a acabar siendo aplicadas las normas. La ventaja de este planteamiento es obvia: estos Estados ya tienen esa potestad, porque precisamente el poder público es la fuente de las potestades, singularmente de las de policía, que, a diferencia de las normativas, no pueden ejercerse de facto por sujetos privados, o no sin violentar los derechos fundamentales al menos... La desventaja de esta opción, sin embargo, también es evidente. Hemos dicho ya, con el ejemplo de los Arbitrajes Internacionales de inversiones, y el CIADI en particular, que no es un gran avance en el derecho global recuperar poderes del Estado. Es más, ante la evidencia del difícil renacimiento del poder del Estado-nación, no resulta razonable intentar imponer las potestades estatales sobre el derecho global, sino al contrario, compatibilizar ambos y resolver razonablemente los conflictos que entre los dos surjan. Todo esto teniendo en cuenta también que un control estatal de los ciudadanos corporativos no puede llevar más que a un caos en el control mismo, al estar descentralizado, y a constantes conflictos diplomáticos según los intereses en juego en el ciudadano corporativo y sus normas. Reforzar el poder de los Estados en particular, ya lo hemos dicho, en el siglo xxi creemos que solo llevará a aislarlos.

Una segunda opción, tal vez más razonable, pasaría por regular los ciudadanos corporativos mediante tratados internacionales ad casum. Desde luego, regular los ciudadanos corporativos con un tratado multilateral general abierto a todos los Estados de la sociedad internacional, incluso aunque fuera 
auspiciado por Naciones Unidas, no nos parece viable: ya hemos dicho que el ciudadano corporativo muta de forma más que ningún otro concepto jurídico que nosotros conozcamos, y someterlo a una regulación general y a priori sin tener en cuenta sus características singulares no nos parece factible. La ventaja de esta opción sería, claro está, una regulación sensible a cada institución reguladora y diplomáticamente pacífica. Entre las desventajas, somos conscientes de que el caos regulatorio persistiría, cuando no se agravaría, si tenemos en cuenta que a cada ciudadano corporativo le seguiría un tratado regulatorio. Pero ¿no es mejor esto que una ley, o peor aún, un reglamento o una circular de cada Estado para cada ciudadano corporativo? Parece evidente que sí.

También es cierto que esta solución conduce irremediablemente a reforzar la posición del derecho internacional público. Esta nos parece mejor opción que reforzar el derecho del Estado, aunque sigue sin ser satisfactoria: el derecho internacional está diseñado para regular la sociedad internacional, no la sociedad global, y por lo tanto sigue situando en su centro los intereses del Estado. Ahora bien, al menos es capaz de aunar las voluntades de sus miembros en un solo tratado por ciudadano corporativo (siendo optimistas...). Aquí es donde consideramos que el arbitraje internacional tiene su más interesante campo de pruebas: el avance en el control de los ciudadanos corporativos puede lograrse introduciendo estos mecanismos de control en las instituciones, por voluntad de todos sus firmantes.

Esto que decimos se asemeja algo a lo que antes explicábamos que argumenta Yackee ${ }^{23}$, aunque ciertamente su propuesta era más intervencionista. Sería preferible que el Estado quedara al margen de intervenir en procedimientos concretos emitiendo opiniones o forzándolas con alguna clase de comité superior, y sí que sometiera a control a la institución en su conjunto, sobre todo para garantizar el cumplimiento con las normas que esta se ha dado a sí misma, con lo que digan los tratados aplicables o con el derecho público global.

Por último, existe una tercera opción para controlar al ciudadano corporativo, o al menos existe en teoría. No podemos ocultar nuestra predilección por esta opción, aunque al mismo tiempo advertimos de su difícil realización. Se trataría del control por el propio derecho global. Lo que nos asalta ahora es evidente: si el derecho global se compone de normas privadas internacionales creadas por ciudadanos corporativos, necesariamente, quien controle al ciudadano corporativo será, poco sorpresivamente, otro ciudadano corporativo. Como es comprensible, esto resulta difícil de asumir a priori. Un ejemplo sencillo puede ser el sistema de recursos interno del CIADI (arts. 50-52 del convenio). Pero vamos a proponer otro ejemplo más complicado.

23 Yackee (2009). 
Expliquémoslo un poco mejor. No se trata sencillamente de que un ciudadano corporativo regule a otro. El derecho global es lo suficientemente abierto e inclusivo como para que, por ejemplo, un Estado y un ciudadano corporativo colaboren en el control de otro, sin perjuicio de que, naturalmente, este último también sea controlado. Así, por ejemplo, un ciudadano corporativo puede dictar normas antiblanqueo o anticorrupción que sean aplicadas a otros ciudadanos corporativos y, ya que no podrá ejecutar las medidas que proponga por falta de potestades para ello (inspección, registro de sedes físicas, examen de documentos, entre otras), podrá ayudarse de la colaboración con un Estado en el cumplimiento de este objetivo, de tal forma que esta entidad privada pueda ejercer, mediante una relación contractual con uno o varios Estados, o con una organización internacional que los represente, funciones propias de una agencia estatal, y al mismo tiempo tener otros objetivos y actividades fuera de esta relación contractual. En virtud de esa misma relación contractual, por ejemplo, los Estados o la organización internacional en cuestión podrían, sin ir más lejos, someter a controles periódicos a este ciudadano corporativo.

Obsérvese lo que se ganaría con semejante trato: en lugar de un control caótico y descentralizado de los ciudadanos corporativos a nivel global, este control se confía a otro ciudadano corporativo muy especializado en esa actividad de control e inspección, por ejemplo, antiblanqueo o anticorrupción, y a su vez recibe esa confianza de una organización internacional que represente a muchos Estados parte, que ya pueden dedicar sus recursos exclusivamente a controlar al ciudadano corporativo que controla. Al mismo tiempo, si un ciudadano corporativo determinado se niega a ser inspeccionado, los Estados que han confiado esas labores al "ciudadano corporativo inspector», por llamarlo así, podrían negar la eficacia de las normas del primero en su territorio. Puede que estemos muy equivocados, pero creemos que así se ganaría eficiencia en el control de los ciudadanos corporativos a nivel mundial, y con menos recursos.

Además, en el caso propuesto, intervienen no solo normas de derecho global, sino también internacional y estatal, lográndose así la hibridación más inextricable y, por lo tanto, la legitimidad de la actuación desde el punto de vista de todas las partes. Este es el camino que, creemos, debe seguir el derecho del siglo XXI. Así, pretendemos mostrar como el derecho global ha llegado para poner orden y sentido en las relaciones de la sociedad global, y no para enturbiarlas, ni para sumir el derecho del Estado en el caos, ni tampoco para perjudicar los valores ya alcanzados por el derecho contemporáneo, que deben servir igualmente de fundamento al derecho global mismo.

Como ejemplos de normas privadas internacionales no asociadas a ninguna institución arbitral en concreto proponemos efectivamente las elaboradas por UNCITRAL (Comisión de Naciones Unidas para el Derecho 
Mercantil Internacional, CNUDMI por sus siglas en español). Como es bien sabido, la Comisión ha creado una ley modelo de arbitraje internacional muy avanzada, cuya primera versión data del año 1985 y cuya reforma corresponde al año 2006, por el momento, y que ha sido adoptada como ley arbitral por muchos Estados miembros, incluida España, mediante la Ley 60/2003, de 23 de diciembre, de Arbitraje. En cualquier caso, lo que nos interesa de esta norma internacional es más bien su posible adopción directa por parte de algunos sujetos. Aclaremos ya que, en todo caso, no hay que confundir las reglas de arbitraje UNCITRAL con la ley modelo, aunque nuestro planteamiento es que ambas pueden usarse como normas privadas internacionales para regular un arbitraje en concreto.

Cabe mencionar, antes de continuar, que la doctrina ha distinguido una serie de normas menores dentro del arbitraje internacional, que ha categorizado como soft law arbitral. Se refieren con este nombre a reglas y directrices de todo tipo que aparecen en el mundo del arbitraje, siendo elaboradas por asociaciones y organizaciones ( $\mathrm{y}$ a veces individuos) con una relevancia menor respecto de las grandes instituciones, y que no tienen vocación de aplicarse obligatoriamente.

El ejemplo más famoso es, desde luego, las reglas de la IBA (International Bar Association), llamadas «Guidelines on Party Representation in International Arbitration on 25 May 2013». Estas directrices se refieren sobre todo a asuntos de conflictos de interés entre las partes y los árbitros, los deberes de comunicación de los árbitros de su propia apariencia de parcialidad, divididos en tres grados, malas prácticas en la información remitida al Tribunal y a la otra parte cuando es preceptivo entregarla y regulación de la concurrencia de testigos y peritos. Estas reglas cuentan con un amplio reconocimiento en el mundo del arbitraje. Hay, además, otras muchas normas sobre estas y otras materias, emitidas también por UNCITRAL y otras organizaciones.

\section{CONCLUSIÓN}

En el presente trabajo hemos intentado demostrar que el Arbitraje internacional de inversiones constituye una rama del derecho global. Este planteamiento aspira a superar la visión del arbitraje como una materia exclusivamente jurídico-privada, y lo concibe como una de las ramas del nuevo orden jurídico global, en el que ocuparía la función de control de la actividad jurídico-pública. De esta forma, hemos expuesto muy brevemente la historia de la institución, para que pueda comprenderse cómo el control del poder público desde una esfera exterior al Estado comienza desarrollándose por la fuerza y en el ámbito del derecho internacional, y evoluciona hacia mecanis- 
mos de control pacíficos y, en último término, privados. Pero, siendo privada la institución encargada de la resolución del conflicto, el objeto de la controversia es el control de la actividad jurídico-pública. De ahí que de facto se produzca un fenómeno de hibridación entre derecho público y derecho privado, característica propia del orden jurídico global. Dicho de otra forma, frente a la creación de normas jurídicas supranacionales como fenómeno más característico del derecho global, es decir, frente al ejercicio de una función normativa, el arbitraje internacional representa el ejercicio de una función de control, y por lo tanto podría calificarse como el reverso del fenómeno jurídico naciente más sobresaliente, el derecho global. 